\title{
The microbiome revolution
}

\author{
Martin J. Blaser \\ Department of Medicine and Microbiology, New York University Langone Medical Center, and New York Harbor Department of Veterans Affairs Medical Center, New York, New York, USA.
}

\begin{abstract}
The collection of bacteria, viruses, and fungi that live in and on the human body, collectively known as the microbiome, has recently emerged as an important factor in human physiology and disease. The gut in particular is a biological niche that is home to a diverse array of microbes that influence nearly all aspects of human biology through their interactions with their host; new technologies are beginning to reveal important aspects of host-microbe interactions. Articles in this Review series address how perturbations of the microbiota, such as through antibiotic use, influence its overall structure and function; how our microbiome influences the impact of infectious agents, such as $C$. difficile; how our microbiome mediates metabolism of xenobiotics; how the microbiota contribute to immunity as well as to metabolic and inflammatory diseases; and the role of commensal microbes in oncogenesis.
\end{abstract}

Until recently, the population of microbes in and on the human body was mostly considered to be vast and largely unknowable. It was referred to as "the normal flora," the collection of plants living with us humans, and was treated as a black box. Now known in its totality as the "microbiome" (1), its function has been debated from the times of Pasteur and Metchnikoff and was believed to be essential or costly to life, respectively (2). We now know that both views are true.

Somehow we believed that the gut microbiome was mostly inert and that, with some notable exceptions, it would not affect the foods or medicines we put in our mouths or on our skin. Also, without much evidence, we believed it to be homeostatic, such that when we would treat bacterial infections with antibiotics, we might perturb it a little, but that everything would just bounce back to normal. We did not think that the microbiome had much relevance to disease, except in the special cases in which it exceeded its confines, such as a ruptured appendix, or was carried by the blood to privileged sites such as heart valves. By and large, considering the overall scope of medical research, the microbiome was a backwater, the domain of some highly specialized scientists and a few generalist pioneers (3-6). But then things began to change.

New technologies, including high-throughput DNA sequencing and bioinformatics, exposed our microscopic interior in ways analogous to the roentgenograms of the prior century. Infusions of concepts from ecology, molecular taxonomy, and evolutionary medicine changed the lens through which we viewed our flora. Systems approaches, once intractable and also anathema to reductionist experimentation, became more popular and fundable. An emphasis on pathogens and virulence mechanisms in microbiologic research was joined by approaches that examined colonization, mutualism, and effects on host phenotypes (7-13).

These new approaches to the microbiome were the start of a revolution. An ancient regime, in which our endogenous microbes were thought to be of only marginal importance, began to tumble.

Conflict of interest: The author acknowledges receipt of research funding from Johnson \& Johnson to his laboratory at New York University and ownership interests in Adamas Pharmaceuticals and Gilead.

Reference information: / Clin Invest. 2014;124(10):4162-4165. doi:10.1172/JCI78366.
The revolution did not come overnight, since the most important considerations had been gaining ground for generations, and were clearly laid out, including vertical transmission, choreographed assembly, interkingdom communication, and symbiosis (1-6). In retrospect, the idea that the organisms we carry must be important is obvious, but this was not the mainstream view of medical science. The overwhelming emphasis was on the human cell, genes, and genome. That is how it should be; the appropriate tools were there, and we indeed are humans.

Yet today medical science also is moving in the direction of the microbiome $(14,15)$, a diverse and rapidly evolving virome (1620), metagenome (14), and the microbial mycome, transcriptome, proteome, and metabolome (21-27). There is increasing evidence that the microbiome and its output (our interactome) touch many, if not most, pathways that affect health, disease, and aging. It is reasonable to propose that the composition of the microbiome and its activities are involved in most, if not all, of the biological processes that constitute human health and disease, as we proceed through our own life cycle (Figure 1).

In this series of Reviews, a group of distinguished scientists who study different aspects of the microbiome have come together to review advances in our knowledge of its workings. Despite its long antecedents, this is a young field; the advanced technologies supporting our interrogations of the microbiome have only been developed in the last decade or so. Scientists are just beginning to outline the problems concerning microbiome variation (28), stability (29), and development $(30,31)$ and the effects of perturbation (32), as well as the consequent interactions with host physiology and pathophysiology $(33,34)$. Epidemiologic studies are beginning to consider the effects of alterations of the microbiome (35, 36). Science begins with observation and moves to experimentation, and the hypotheses tested lead to new ideas and ultimately to approaches and products that improve human health.

These future products might involve the introduction of therapeutic microbes, either singly or in consortia. Large consortia are currently being used in the form of the fecal microbial transplant for treatment of Clostridium difficile infection (CDI) (37). Alternatively, chemicals (prebiotics) that serve as substrates for favored 


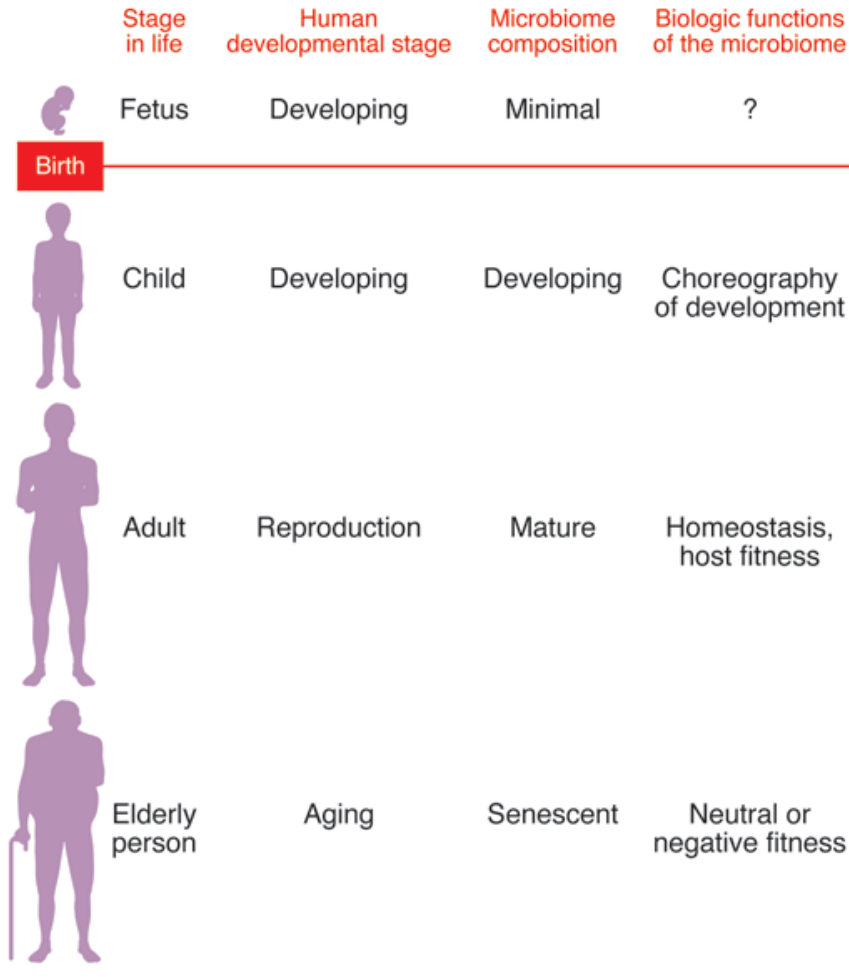

microbes or favored metabolic pathways could be used to effect changes in host-microbial interactions (38). Study of the microbiome may allow us to harness specific microbial constituents to affect physiology. Just as the metabolic capability of the lethal pathogen Clostridium botulinum has been harnessed to correct strabismus, muscle spasm, and a myriad of cosmetic problems, scientists will unearth new compounds developed over the eons by our microscopic cell biologists to serve their own purposes.

Another area of discovery is the identification of the host compounds that are part of the transduction of signals from microbes to their hosts. Just as studying the pathogen Vibrio cholerae helped us clarify the roles of adenyl cyclase and cAMP in human biology, study of commensals, such as the helicobacters, clostridia, and bacteroides, among countless others, will help us understand signaling within epithelial cells and to innate and adaptive immune cells, as well as the ontogeny of their responses $(31,33,34)$. This is a particularly exciting frontier because it suggests that one day we will have sufficient knowledge to manipulate immune responses in the short and long term using specific (probiotic) microbes or prebiotics that persons could ingest or apply to affected areas.

A listing of important topics highlighted by the authors in this series can be summarized in the following sections. I have taken the liberty of framing the papers in terms of seven large biological questions pertinent to human health.

\section{Perturbation of the microbiota to understand its fundamental structure}

Modi et al. (39) explore the relationship of antibiotics to the microbiota, not as therapeutic agents that control infections, but rather from the context of their role as signaling molecules that affect the ecology of the microbiome. An important focus is how antibiotic
Figure 1. Interpretation of the major biological roles of the microbiome in health and disease. In this global model, the microbiome affects physiologic functions at each life stage as a participant in life cycle events and processes. During early life development, as the microbiome transforms, it gains diversity and complexity, maturing into an adult-type pattern. This transformation occurs in parallel with host metabolic, immunologic, and cognitive development and undoubtedly contributes to normal physiology. Perturbation could thus have important deleterious consequences. After reproductive life wanes, the selection on the microbiota differs, with important consequences for diseases. I hypothesize that the changed selection fuels the descent into the degenerative and neoplastic diseases of aging.

exposures affect colonization resistance, the property of a defined ecosystem to oppose the entry of a pathogen. A related concept is the effects of the antibiotic on resilience of the ecosystem, the ability for the composition of the microbiota to bounce back after perturbation. This can be considered at the level of ecosystem, community, species, strain, and/or metabolite; all are germane. Another emerging concept is the importance of antibiotic exposure on the horizontal transfer of resistance genes originating in commensals. These concepts are relevant to the tens of millions of people who receive an antibiotic once a year or more, exposures generally regarded as inconsequential beyond the period of exposure, a view that is almost certainly incorrect (40-42).

\section{Acute clinical consequences of perturbing the microbiota}

Just about every perturbation can be considered worthy of study, but some are more obvious than others, such as the colitis due to CDI that most typically follows antibiotic exposure. Seekatz and Young discuss potential relationships between C. difficile, a human commensal that has become a pathogen, and other members of the human gut microbiota (43). They highlight an important field of inquiry: are some of the taxa that are inversely correlated with CDI potential inhibitors, i.e., organisms whose properties can be harnessed for either protection or for treatment of the disordered ecosystem (dysbiosis)? Similarly, current research has identified particular chemical entities that may promote or inhibit $C$. difficile germination and growth. CDI is epidemic, and finding medical solutions to the problem is important a priori, but by using the interventions uncovered as a model, similar approaches can be developed to prevent other infections or to curtail colonization by microbes that are unfavorable.

\section{Microbiota-mediated modification of pharmaceuticals}

The cumulative exposure of humans to pharmaceuticals, overthe-counter agents, and other xenobiotics is enormous. Carmody and Turnbaugh focus on the direct and indirect effects of the microbiota on the metabolism of such chemicals (44). This is a critical field of clinical investigation because there is substantial variation in the pharmacokinetics of most commonly used drugs, and large differences in the responses of ill persons to therapeutics. Similarly, it is becoming clear than many of the toxic effects of particular drugs and the detoxification of others are due to microbial interactions. Identification of the key microbial players and of the relevant metabolic pathways is certain to become a major area 
of inquiry. Over time, we should be able to establish the general principles governing drug metabolism by the microbiota in relation to interhost diversity. Predictably, this will become a new discipline within pharmacology (45).

\section{Microbial contributions to the development of immunity}

Babies are born with innate immunity and with a pathway toward the acquisition of adaptive immunity, but they begin life immunologically naive (46). Surana and Kasper explore how the microbiome contributes to the development and homeostasis of the immune system (47). Several microbes have well-characterized effects on particular immune functions, but these are just the tip of the iceberg. We can imagine that the "probiotic" administration of specific microbes will be useful in the future when, for example, we wish to modulate autoimmunity via the upregulation of Th17 cells, or to combat immunodeficiency through the recruitment of Th1 cells. There must be much investigation of the organisms that produce strong monochromatic effects, and the mechanisms by which they communicate with host cells and regulate specific immune functions (31), prior to the development of true "probiotic" therapies. As reported by Surana and Kasper (47), location, timing, and context are important co-variables that affect microbial interactions with immunity.

\section{Interkingdom metabolic exchange and disease}

The human microbiome has a wide range of metabolic activities that stand to have a substantial impact on human physiology and disease. The ability of colonic bacteria to ferment nondigestible fiber into energy substrate (short-chain fatty acids) for the benefit of both microbes and the host is well established (48). Tang and Hazen explore how intestinal microbial metabolism exerts effects on host metabolism, including and culminating in atherosclerotic cardiovascular disease (ASCVD) (49). These findings are important to our understanding of ASCVD in human aging and mortality, but also serve as a paradigm for the extensively linked interactions between the capabilities of our commensals (that dine at our table) and our own metabolism. In their model, using trimethylamine-N-oxide (TMAO) as an indicator molecule, they advance the concept that gut metabolites might be analogous to hormones, with substantial effects at sites distant from their production. In such a manner, the microbiota transform the energy substrate of our diets into the hormonal messengers themselves or into their precursors, or conversely, into their antagonists. The possibilities and applications are legion.

\section{Microbial contributions to the pathogenesis of chronic inflammatory diseases}

Among the most troubling and persistent diseases of modern peoples are the chronic disorders in which tissues are damaged by inflammation that is out of control. Dalal and Chang focus on a well-known clinical problem, inflammatory bowel disease (IBD), as an example of a dysbiosis that may lead to illness (50). They consider evidence that host genotypes select for particular microbial compositions as a part of their phenotypic expression, and they provide a context for what might be an important connection between the known genetic basis for IBD and the microbiome.
Similarly, they speculate that some of the known dietary effects on IBD may be mediated at least in part on selection of particular members of the microbiota. Critically, the differences in commensal composition that are seen in individuals with IBD compared with controls may reflect selection by the disease itself or in its treatment, rather than an actual predisposition. Clinical investigators must be careful about reaching appropriate etiologic conclusions for patients studied in the midst of their disease.

\section{Costs of commensalism: inflammation and oncogenesis}

An important question concerns the biological cost to the host of carrying an extensive microbiota. Sears et al. (51) study Bacteroides fragilis, a well-known human pathogen that causes extra-intestinal infections but is also a probable symbiont. The production of a zinc-dependent metalloprotease separates these organisms into two classes, with the producers being associated with more aggressive local biological activities, including the induction of inflammation and, perhaps, cancer. These studies provide a model of oncogenic contributions by commensals, similar to the role of Helicobacter pylori in gastric carcinogenesis (52). Being able to classify the microbiota on the basis of single molecules that define pathological outcomes to a high degree (e.g., the B. fragilis enterotoxin) provides another level of understanding of the host interactions with the microbiota.

\section{Perspective and future directions}

The topics covered in this Review series are examples of current outstanding research on the biology of the human microbiome, but they are by no means inclusive. Emerging disciplines concern the development of metabolism, immunity, and cognition and reproductive capabilities and disorders related to aberrant development $(42,53)$, among other exciting areas, with new insights about the very nature of heredity (30). Exploration of the virome, its diversification and evolution, and its interactions with prokaryotes and with the host $(54,55)$ will be a particularly interesting frontier. Similarly, the early studies providing evidence of a linkage between the gut microbiome and neural processes, including development, cognition, and emotion (56-58), indicates the range of possibilities. Development of open-source software tools makes the technology accessible to most investigators $(59,60)$. The horizons are broad, with exciting developments ahead.

\section{Acknowledgments}

This work was supported in part by the NIH (grants UH2AR057506, R01GM62370, and T-R01DK090989), US Army Medical Research grant W81XWH-11-1-0739, and the Juvenile Diabetes Research Foundation. The author thanks the Diane Belfer Fund, the Knapp and Ziff Foundations, and an anonymous donor for their support of his work on the human microbiome.

Address correspondence to: Martin J. Blaser, Muriel and George Singer Professor of Medicine, Professor of Microbiology, Director, Human Microbiome Program, Department of Medicine and Microbiology, New York University Langone Medical Center, 550 First Avenue, Bellevue CD 689, New York, New York 10016, USA. Phone: 646.501.4386; E-mail: Martin.Blaser@nyumc.org. 
1. Lederberg J. Infectious history. Science. 2000;288(5464):287-293.

2. Mackowiak PA. The normal microbial flora. N Engl J Med.1982;307(2):83-93.

3. Rosebury T. Life On Man. New York, New York, USA: Viking Adult; 1969.

4. Bohnoff M, Drake B, Miller C. Effect of streptomycin on susceptibility of intestinal tract to experimental Salmonella infection. Proc Soc Exp Biol Med. 1954;86(1):132-137.

5. Dubos RJ, Savage DC, Schaedler RW. The indigenous flora of the gastrointestinal tract. Dis Colon Rectum. 1967;10(1):23-34.

6. Savage DC. The microbial flora in the gastrointestinal tract. Prog Clin Biol Res. 1981;77:893-908.

7. Beard A, Blaser MJ. The ecology of height: the effect of microbial transmission on human height. Perspect Biol Med. 2002;45(4):475-498.

8. Bäckhed F, et al. The gut microbiota as an environmental factor that regulates fat storage. Proc Natl Acad Sci U S A. 2004;101(44):15718-15723.

9. Blaser MJ. Who are we? Indigenous microbes and the ecology of human diseases. EMBO Rep. 2006;7(10):956-960.

10. Dethlefsen L, McFall-Ngai M, Relman D. An ecological and evolutionary perspective on human-microbe mutualism and disease. Nature. 2007;449(7164):811-818.

11. Blaser MJ, Kirschner D. The equilibria that permit bacterial persistence in human hosts. Nature. 2007;449(7164):843-849.

12. Barton ES, et al. Herpesvirus latency confers symbiotic protection from bacterial infection. Nature. 2007;447(7142):326-329.

13. Fukuda $S$, et al. Bifidobacteria can protect from enteropathogenic infection through production of acetate. Nature. 2011;469(7331):543-547.

14. The Human Microbiome Project Consortium. Structure, function and diversity of the healthy human microbiome. Nature. 2012;486(7402):207-214.

15. The Human Microbiome Project Consortium. A framework for human microbiome research. Nature. 2012;486(7402):215-221.

16. Reyes A, et al. Viruses in the faecal microbiota of monozygotic twins and their mothers. Nature. 2010;466(7304):334-338.

17. Minot S, et al. The human gut virome: inter-individual variation and dynamic response to diet. Genome Res. 2011;21(10):1616-1625.

18. Pride DT, et al. Evidence of a robust resident bacteriophage population revealed through analysis of the human salivary virome. ISME J. 2011;6(5):915-926.

19. Minot S, Bryson A, Chehoud C, Wu GD, Lewis JD, Bushman FD. Rapid evolution of the human gut virome. Proc Natl Acad Sci U S A. 2013;110(30):12450-12455.

20. Wylie KM, Mihindukulasuriya KA, Zhou Y, Sodergren E, Storch GA, Weinstock GM. Metagenomic analysis of double-stranded DNA viruses in healthy adults. BMC Biol. In press.

21. Yoshimoto S, et al. Obesity-induced gut microbial metabolite promotes liver cancer through senescence secretome. Nature. 2013;499(7456):97-101.
22. Joyce SA, et al. Regulation of host weight gain and lipid metabolism by bacterial bile acid modification in the gut. Proc Natl Acad Sci U S A. 2013;111(20):7421-7426.

23. Davey KJ, et al. Antipsychotics and the gut microbiome: olanzapine-induced metabolic dysfunction is attenuated by antibiotic administration in the rat. Transl Psychiatry. 2013;3:e309.

24. David LA, et al. Diet rapidly and reproducibly alters the human gut microbiome. Nature. 2014;505(7484):559-563.

25. Ridaura V, et al. Gut microbiota from twins discordant for obesity modulate metabolism in mice. Science. 2013;341(6150):1241214.

26. Le Chatilier E, et al. Richness of human gut microbiome correlates with metabolic markers. Nature. 2013;500(7464):541-546.

27. Findley K, et al. Topographic diversity of fungal and bacterial communities in human skin. Nature. 2013;498(7454):367-370.

28. Kraal S, Abubuker S, Kota K, Fischbach MA. The prevalence of species and strains in the human microbiome: a resource for experimental efforts. PLoS One. 2014;9(5):e97279.

29. Sharon I, Morowitz MJ, Thomas BC, Costello EK, Relman DA, Banfield JF. Time series community genomics analysis reveals rapid shift in bacterial species, strains, and phage during infant gut colonization. Genome Res. 2013;23(1):111-120.

30. Koren O, et al. Host remodeling of the gut microbiome and metabolic changes during pregnancy. Cell. 2012;150(3):470-480.

31. Chung $\mathrm{H}$, et al. Gut immune maturation depends on colonization with a host-specific microbiota. Cell. 2012;149(7):1578-1593.

32. Modi SR, Lee HH, Spina CS, Collins JJ. Antibiotic treatment expands the resistance reservoir and ecological network of the phage metagenome. Nature. 2013;499(7457):219-222.

33. Ivanov II, et al. Induction of intestinal Th17 cells by segmented filamentous bacteria. Cell. 2009;139(3):485-498.

34. Wu HJ, et al. Gut-residing segmented filamentous bacteria drive autoimmune arthritis via Thelper 17 cells. Immunity. 2010;32(6):815-827.

35. Trasande L, Blustein J, Liu M, Corwin E, Cox LM, Blaser MJ. Infant antibiotic exposures and early life body mass. Int JObes (Lond). 2013; 37(1):16-23.

36. Blustein J, et al. Caesarian delivery and child adiposity. Int JObes (Lond). 2013;37(7):900-906.

37. van Nood E, et al. Duodenal infusion of donor feces for recurrent Clostridium difficile. $N$ Engl J Med. 2013;368(5):407-415.

38. Antharam VC, et al. Intestinal dysbiosis and depletion of butyrogenic bacteria in Clostridium difficile infection and nosocomial diarrhea. JClin Microbiol. 2013;51(9):2884-2892.

39. Modi SR, Collins JJ, Relman DA. Antibiotics and the gut microbiota. J Clin Invest. 2014;124(10):4212-4218.

40. Hviid A, Svanstrom H, Frisch M. Antibiotic use and inflammatory bowel diseases in childhood. Gut. 2011;60(1):49-54.

41. Kozyrskyj AL, Ernst P, Becker AB. Increased risk of childhood asthma from antibiotic use in early life. Chest. 2007;131(6):1753-1759.
42. Cox LM, et al. Altering the intestinal microbiota during a critical developmental window has lasting metabolic consequences. Cell. 2014;158(4):705-721.

43. Seekatz AM, Young VB. Clostridium difficile and the microbiota. J Clin Invest. 2014; 124(10):4182-4819.

44. Carmody RN, Turnbaugh PJ. Host-microbial interactions in the metabolism of therapeutic and diet-derived xenobiotics. JClin Invest. 2014;124(10):4173-4181.

45. Wallace BD, et al. Alleviating cancer drug toxicity by inhibiting a bacterial enzyme. Science. 2010;330(6005):831-835.

46. Marchant A, Goldman M. T cell-mediated immune responses in human newborns: ready to learn? Clin Exper Immunol. 2005;141(1):10-18.

47. Surana NK, Kasper DL. Deciphering the tête-àtête between the microbiota and the immune system. JClin Invest. 2014;124(10):4197-4203.

48. Topping DL, Clifton PM. Short-chain fatty acids and human colonic function: roles of resistant starch and nonstarch polysaccharides. Physiol Rev. 2001;81(3):1031-1064

49. Tang WHW, Hazen SL. The contributory role of gut microbiota in cardiovascular disease. J Clin Invest. 2014;124(10):4204-4211.

50. Dalal SR, Chang EB. The microbial basis of inflammatory bowel diseases. J Clin Invest. 2014;124(10):4190-4196.

51. Sears CL, Geis AL, Housseau F. Bacteroides fragilis subverts mucosal biology: from symbiont to colon carcinogenesis. JClin Invest. 2014;124(10):4166-4172.

52. Kamangar F, et al. Opposing risks of gastric cardia and noncardia gastric adenocarcinomas associated with Helicobacter pylori seropositivity. J Natl Cancer Inst. 2006;98(20):1445-1452.

53. Cho I, et al. Antibiotics in early life alter the murine colonic microbiome and adiposity. Nature. 2012;488(7413):621-626.

54. Reyes A, Wu M, McNulty NP, Rohwer FL, Gordon JI. Gnotobiotic mouse model of phage-bacterial host dynamics in the human gut. Proc Natl Acad Sci U S A. 2013;110(50):20236-20241.

55. Barr JJ, et al. Bacteriophage adhering to mucus provide a non-host-derived immunity. Proc Natl Acad Sci U S A. 2013;110(26):10771-10776.

56. Mayer EA. Gut feelings: the emerging biology of gut-brain communication. Nat Rev Neurosci. 2011;12(8):453-466.

57. Bravo JA, et al. Ingestion of Lactobacillus strain regulates emotional behavior and central GABA receptor expression in a mouse via the vagus nerve. Proc Natl Acad Sci U S A. 2011;108(38):16050-16055.

58. Diaz Heijtz R, et al. Normal gut microbiota modulates brain development and behavior. Proc Natl Acad Sci U S A. 2011;108(7):3047-3052.

59. Caporaso JG, et al. QIIME allows analysis of highthroughput community sequencing data. Nat Methods. 2010;7(5):335-336.

60. Schloss PD, et al. Introducing mothur: opensource, platform-independent, community-supported software for describing and comparing microbial communities. Appl Environ Microbiol. 2009;75(23):7537-7541. 